\title{
Factores de resiliencia escolar y el éxito micro empresarial en el Distrito de Huacho
}

\section{Factors of school resilience and micro business business success in the Huacho District}

\author{
Cesar Marcelino Mazuelos Cardoza', Carlos Máximo Gonzales Añorga'
}

\begin{abstract}
RESUMEN
Objetivo: Demostrar que los factores de resiliencia en el educando influyen significativamente en el éxito micro empresarial. Material y Métodos: Fue una investigación descriptivo-explicativo-correlacional y causal, tomando una muestra de 384 microempresarios. Resultados: Un mayoritario $52,1 \%$ manifestaron estar de acuerdo que su época de escolar estuvo marcada por la resiliencia, un 41,4\% manifestó estar de acuerdo que durante sus años de estudios tenía un alto nivel de capacidad reflexiva, un porcentaje relativamente bajo equivalente al $22,9 \%$ manifestó estar de acuerdo que vivió un ambiente de alta cohesión familiar. Conclusión: La capacidad reflexiva está presente en muchas personas en edad escolar, la utilización racional de la mente humana está presente en las personas con un mínimo de pensamiento formal, las capacidades intelectuales no solamente son manifiestas en personas con experiencia en la vida, sino también están inherentes en personas con poca edad.
\end{abstract}

Palabras Clave: Resiliencia, desarrollo personal, capacidad intelectual, responsabilidad empresarial.

\begin{abstract}
Objective: To demonstrate that the resilience factors in the student significantly influence the micro business success. Material and methods: It was a descriptive-explanatory-correlational and causal investigation, taking a sample of 384 micro entrepreneurs. Results: A majority $52,1 \%$ said they agreed that their school age was marked by resilience, $41,4 \%$ said they agreed that during their years of study they had a high level of reflective capacity, a relatively low percentage equivalent to $22,9 \% \mathrm{He}$ said he agreed that he lived an environment of high family cohesion. Conclusion: The reflective capacity is present in many people of school age, the rational use of the human mind is present in people with a minimum of formal thinking, intellectual abilities are not only manifest in people with experience in life, but also They are inherent in people of young age.
\end{abstract}

Keywords: Resilience, personal development, intellectual capacity, corporate responsibility

\section{INTRODUCCIÓN}

La presente investigación referida a la presencia de factores de resiliencia en educandos se justifica porque brinda la oportunidad de dar a conocer una realidad existente en nuestro medio y a su vez generar estímulos para tomar decisiones pertinentes a las acciones a desarrollar, debiendo ser estas acciones las más acordes a las que el educando resiliente necesita. Por supuesto, ello depende mucho de las personas que tienen a su cargo la gerencia de las instituciones educativas, las mismas que desde los más altos niveles deben promover programas que permitan una orientación adecuada.

\section{Antecedentes}

Peralta, Ramírez y Buitrago (2005) desarrollaron una investigación de tipo correlacional cuyo objetivo fue determinar si existen factores resilientes asociados al rendimiento académico en estudiantes universitarios. Evaluaron a 345 estudiantes, distribuidos en dos grupos: bajo rendimiento y alto rendimiento, entre los 16 y 38 años de edad. El instrumento que utilizaron fue el cuestionario de resiliencia para estudiantes universitarios (CRE-U). Sus resultados encontraron que hay diferencias estadísticamente significativas de los factores resilientes entre los estudiantes con alto y bajo rendimiento académico, y este último grupo es el que se encuentra en mayor riesgo debido a que existen menos factores que se asocian a él.

Villalta (2010), realizó un estudio para analizar la relación entre factores de resiliencia y el rendimiento académico en alumnos adolescentes de establecimientos educativos ubicados en contextos de alta vulnerabilidad social. Se trata de un estudio correlacional realizado con una población de 437 alumnos de Educación Media de la Región Metropolitana de Chile. Diseñó y aplicó un cuestionario para determinar el nivel de calidad de vida y factores de riesgo de los adolescentes. La resiliencia lo midió con la escala SVRES creada para población chilena.

Sus resultados indicaron que la relación entre resiliencia y logros de aprendizaje se fortalece en dos situaciones de adversidad que reportan los adolescentes: El divorcio o separación de sus padres y el embarazo propio o de la pareja.

Velásquez y Montgomery (2009) realizaron un estudio correlacional entre resiliencia y depresión en instituciones educativas de Lima, con alumnos del tercero al quinto de secundaria, de género masculino y femenino, de los cuales una parte no ha participado en actos violentos y otra sí. Utilizaron como instrumentos para la evaluación la escala de resiliencia y el inventario de depresión en la adolescencia, determinaron que existen correlaciones positivas en los componentes internos de ambas pruebas, mientras que al correlacionarlas entre ellas la relación es más diversa. También identificaron diferencias entre los alumnos violentos y no violentos en cuanto a los factores de confianza, autoritarismo e ideas suicidas. Asimismo, en la comparación inter sexo, se identificaron diferencias entre mujeres y varones respecto al grado de control emocional, toma de decisiones, autocrítica, propensión al llanto y otras variables. Por lo tanto esta investigación puede servir de plataforma para emprender programas mayores, con el propósito de minimizar la indiferencia existente en el ámbito educativo respecto a la presencia de educandos resilientes en los planteles educativos y contribuir a elevar la calidad educativa de los mismos.

\section{MATERIALES Y MÉTODOS}

\section{Diseño metodológico:}

Tipo de la Investigación:

El presente estudio es una Investigación Aplicada, ya que se busca resolver un problema y encontrar respuestas a preguntas específicas.

Nivel o diseño específico de la investigación:

La presente investigación es de nivel descriptivo correlacional Causal 


\section{Población y Muestra}

a) Población:

La población estudiada estuvo comprendida por todos aquellos micro empresarios del distrito de Huacho, que en la actualidad está compuesta por un total de 4,436 microempresarios.

\section{b) Muestra:}

Para la obtención de la muestra se aplicó el muestreo probabilístico (aleatorio simple), la misma que se obtuvo mediante el procesamiento de la siguiente fórmula estadística:

$$
n=\frac{N \times P \times Q \times Z^{2}}{E^{2} \times(N-1)+P \times Q \times Z^{2}}
$$

Donde:

$\mathbf{n}=$ Tamaño de la muestra

$\mathbf{N}=$ Tamaño de la población.

$\mathbf{E}=0,05$ (nivel de error $5 \%$ )

$\mathbf{P}=0,5 \%$ (probabilidad de 50\%)

$\mathbf{Q}=0,5 \%$ (probabilidad de $50 \%$ )

$\mathbf{Z}=1,96$ (95\% de nivel de confianza)

Siendo el tamaño de muestra 384

Técnicas de recolección de datos:

Fuentes de recolección de datos:

La información que se recolectó para la investigación, principalmente fue mediante la encuesta, que es una fuente primaria muy importante para este tipo de estudio.

Instrumentos de recolección de datos:

Para el estudio se utilizó del cuestionario como herramienta principal en la recolección de los datos.

En relación a la interrogante si el encuestado vivió un ambiente de alta cohesión familiar, los resultados obtenidos se mostraron de la siguiente manera: Un porcentaje relativamente bajo equivalente al 22,9\% manifestó estar de acuerdo que vivió un ambiente de alta cohesión familiar, seguido de un $15,9 \%$ que expresó estar totalmente de acuerdo. Paralelamente existe un mayoritario $25,8 \%$ que respondió no estar de acuerdo ni en desacuerdo, mientras que un $22,1 \%$ y un $13,3 \%$ manifestaron estar en desacuerdo o totalmente en desacuerdo respectivamente. Estos resultados son indicios que los encuestados no vivieron en un hogar armonioso necesariamente, lo cual podría haber un factor complementario de mayor adversidad y resiliencia. Complementariamente se puede indicar que en porcentaje de los casos equivalente al $38.8 \%$, respondieron que pese a las adversidades en sus años de escolar si vivieron en un ambiente de alta cohesión familiar.

Técnicas para el procedimiento de la información:

Se utilizó el programa SPSS o Paquete Estadístico para Ciencias Sociales (Statistical Package for the Social Sciences) en su versión 21,0 para el procesamiento de los datos. Para la contrastación de hipótesis se tuvo en cuenta que las variables son cualitativas y el cuestionario propone respuestas tipo ordinal, por lo que para el coeficiente de correlación se utilizó el estadístico Tau-b de Kendall.

Hipótesis General:

"Los Factores de Resiliencia en el Educando influyen significativamente en el éxito Micro empresarial".

Hipótesis Específicas:

a) El nivel de capacidad reflexiva como factor de resiliencia, incide en las oportunidades de negocio b) El nivel de la capacidad intelectual como factor de resiliencia, influye en la capacidad empresarial

c) La cohesión familiar como factor de resiliencia repercute en la adecuada Gestión Empresarial

d) El nivel de apoyo externo como factor de resiliencia, repercute en la magnitud del Desarrollo Empresarial.

\section{RESULTADOS}

Tabla 1

Género del(a) empresario(a) encuestado(a): El resultado respecto al género del empresario (a) encuestada fue el siguiente

\begin{tabular}{lccc} 
& Frecuencia & $\begin{array}{c}\text { Porcentaje } \\
\%\end{array}$ & $\begin{array}{c}\% \\
\text { Acumulado }\end{array}$ \\
\hline Masculino & 246 & 64,1 & 64,1 \\
Femenino & 138 & 35,9 & 100,0 \\
\hline Total & 384 & 100,0 & \\
\hline
\end{tabular}

La información obtenida demuestra que el microempresario en la ciudad de Huacho de forma mayoritaria pertenece al género masculino, aunque ello no necesariamente refleja en términos generales tal porcentualidad, ya que en este estudio se considera solamente al segmento de microempresarios resilientes. $64,1 \%$ de los microempresarios pertenecen al género masculino, mientras que el $35,9 \%$ pertenecen al género femenino. Ello aparentemente contrastaría con otros estudios que demostraron que pertenecer al género femenino facilitó mucho la resiliencia.

Tabla 2

Época escolar marcada por la Resiliencia: A la pregunta: ¿Considera usted que su época de escolar estuvo marcada por la resiliencia?

\begin{tabular}{lccc}
\hline & & $\begin{array}{c}\text { Porcentaje } \\
\%\end{array}$ & $\begin{array}{c}\% \\
\text { Acumulado }\end{array}$ \\
& Frecuencia & $\%, 8$ & 45,8 \\
\hline $\begin{array}{l}\text { Totalmente de } \\
\text { acuerdo }\end{array}$ & 176 & 45,8 & \\
De acuerdo & 200 & 52,1 & 97,9 \\
Ni de acuerdo & 5 & 1,3 & 99,2 \\
ni en & & & \\
desacuerdo & & & \\
En desacuerdo & 1 & 0,3 & 99,5 \\
Totalmente en & 2 & 0,5 & 100,0 \\
desacuerdo & & & \\
\hline Total & 384 & 100,0 & \\
\hline
\end{tabular}

Los resultados de esta interrogante demostraron que un mayoritario $52,1 \%$ manifestaron estar de acuerdo que su época de escolar estuvo marcada por la resiliencia, sin embargo, un $45,8 \%$ manifestó estar totalmente de acuerdo que su época escolar estuvo marcada por la resiliencia, ello indica que un contundente $97,9 \%$ del total de los encuestados manifestaron haberse desenvuelto en resiliencia durante su época escolar. El restante $2,1 \%$ de encuestados manifestó que su época escolar no estuvo marcada por la resiliencia. Cabe agregar aqui, que este ultimo número de encuentados manifestaron que su época escolar no estuvo marcada por la resiliencia no implica que jamas tuvieron adversidades, si no mas bien que 
las mismas no fueron permanentes, pero que si las vivieron.

\section{Tabla 3}

Nivel de capacidad reflexiva: a la pregunta: ¿Considera que en su época de escolar usted tenía un alto nivel de capacidad reflexiva?

$\%$

\begin{tabular}{lccc} 
& Frecuencia & $\%$ & Acumulado \\
\hline $\begin{array}{l}\text { Totalmente de } \\
\text { acuerdo }\end{array}$ & 121 & 31,5 & 31,5 \\
De acuerdo & 159 & 41,4 & 72,9 \\
Ni de acuerdo & 50 & 13,0 & 85,9 \\
ni en & & & \\
desacuerdo & 46 & 12,0 & 97,9 \\
En desacuerdo & 8 & 2,1 & 100,0 \\
Totalmente en & & & \\
desacuerdo & 384 & 100,0 & \\
\hline Total & & & \\
\hline
\end{tabular}

Cuando a los encuestados se les preguntó respecto a su nivel de capacidad reflexiva durante sus años de escolar, los resultados fueron los siguientes: Un alto porcentaje correspondiente al 41,4\% manifestó estar de acuerdo que durante sus años de estudios tenía un alto nivel de capacidad reflexiva, seguido de un $31,5 \%$ que expresó estar totalmente de acuerdo que durante su época de escolar tenía un alto nivel de capacidad reflexiva, sin embargo un $13 \%$ de encuestados respondió no estar de acuerdo ni en desacuerdo, seguido de un $12 \%$ que respondió estar en desacuerdo que durante sus años de estudios tenía un alto nivel de capacidad reflexiva, seguido de un minoritario $2,1 \%$ que expresó estar totalmente en desacuerdo. Estos resultados demuestran que muchos microempresarios sienten o piensan que a pesar de las adversidades que vivieron durante sus años de estudios escolares, tenían un alto nivel de capacidad reflexiva que les hacía notar la realidad de ese entonces.

\section{Tabla 4.}

Nivel de cohesión familiar: a la pregunta: ¿Considera que en su época de escolar usted vivía un ambiente de alta cohesión familiar?

Frecuencia

\begin{tabular}{|lccc}
\hline $\begin{array}{l}\text { Totalmente de } \\
\text { acuerdo }\end{array}$ & 61 & 15,9 & 15,9 \\
\hline $\begin{array}{l}\text { De acuerdo } \\
\begin{array}{l}\text { Ni de acuerdo ni } \\
\text { en desacuerdo }\end{array}\end{array}$ & 98 & 22,9 & 38,8 \\
$\begin{array}{l}\text { En desacuerdo } \\
\begin{array}{l}\text { Totalmente en } \\
\text { desacuerdo }\end{array}\end{array}$ & 85 & 25,8 & 64,6 \\
\hline Total & 384 & 13,3 & 100,0 \\
\hline
\end{tabular}

\section{CONCLUSIÓN}

El presente estudio de investigación ha tenido como principal objetivo demostrar que muchas personas en edad escolar que estuvieron aquejadas de muchas privaciones materiales que, de no haber sido así, podría haberle facilitado la obtención de mayores y mejores resultados en su mundo académico; sin embargo, pese a estas privaciones, tales personas siendo ya adultas, han logrado un significativo éxito en sus actividades micro empresariales.

El grupo de microempresarios que colaboraron mediante el desarrollo de la encuesta que se les entregó para el presente estudio de investigación, ha sido bastante asequible y colaborador, teniendo en consideración que el común denominador de todos ellos es el hecho de que pasaron por una etapa de resiliencia en su vida escolar. Para ello, antes de la encuesta necesariamente se tuvo un pequeño dialogo donde se les esclareció todo tipo de interrogantes y dudas por parte de ellos, sobre todo las nociones de resiliencia, que no es un término muy usual en el común ciudadano. La mayoría de ellos ha encajado dentro de los principales factores de resiliencia que el estudio consideró, tales como: el nivel de capacidad reflexiva, la capacidad intelectual, la cohesión familiar y el nivel de apoyo externo que pudieron haber percibido durante sus años de escolar.

En términos generales, el grupo de estudio se adecua sustancialmente a lo que la investigación ha pretendido demostrar. En forma mayoritaria los microempresarios aceptaron que perciben haber tenido una alta capacidad reflexiva en los años en que eran escolares, lo que implica que la privación en su dimensión material, no es un obstáculo cuando la persona, a pesar de su edad escolar, tiene capacidad de reflexión y su dimensión cerebral, desde el punto de vista psíquico, se expande en la meditación del mundo que lo rodea. Respecto al factor de capacidad intelectual, muchos de las personas que colaboraron con la investigación han corroborado que desde temprana edad se pueden desarrollar las habilidades de la estructura del intelecto tales como el saber comprender las cosas, el tener buena memoria, saber solucionar problemas, saber tomar decisiones y tener creatividad, aun cuando el percato de dichas variables se dio en plena edad escolar. Por otro lado y a pesar de que los resultados no muestran una contundente mayoría, la variable cohesión familiar, da cuenta que en alguna medida, el interés familiar, la ayuda y el apoyo mutuo en familia puede ser pertinente para que las personas no se dejen avasallar por las adversidades que puedan tener en los años tempranos de la vida; en palabras más sencillas, se puede entender que el niño en edad escolar que siente apoyo de la familia, en su nivel de reflexión, cavilará que la familia no puede ofrecerle mayores niveles materiales, pero entiende que si pudiera lo haría. Por último, en cuanto a la variable referida al nivel de apoyo externo, ésta básicamente se refiere al apoyo moral, e incluso material, que pudo haber tenido el niño en su época escolar por parte de alguna persona cercana, pero no de su inmediato ambiente familiar, como por ejemplo un profesor, un vecino o tal vez algún filántropo cercano al medio en que se desenvolvió; el estudio nos ofrece un resultado optimista en cuanto al nivel de apoyo externo obtenido por quienes formaron parte de la presente investigación.

Ahora bien, la investigación ha sido congruente con autores como Werner (1989) y Garmezy (1991), quienes dan cuenta de factores de resiliencia que se observan en niños de temprana edad y están relacionados con las variables de las que se ha comentado en el párrafo anterior, sin embargo, el estudio difiere de algunas investigaciones que observaron que pertenecer al género femenino es una variable protectora tal como lo sostienen Kotliarenko, Cáceres y Fontecilla (1997); los mismos autores citan a Rutter (1991) quien se refiere al género masculino como una variable que genera mayor vulnerabilidad al riesgo. En tales circunstancias es necesario establecer que el presente estudio tiene un porcentaje de microempresarios que pertenecen al género femenino equivalente a un trentaiseis por ciento, términos nada desdeñables si enfatizamos el carácter del género al que se hace referencia. 
Por lo anteriormente referido, cabe la posibilidad de sugerir nuevos estudios de investigación para corroborar si efectivamente el género femenino tiene menores riesgos cuando se manifiesta factores de resiliencia, ya que el presente estudio no trata de demostrar qué género es más vulnerable a los riesgos cuando se vive en resiliencia; complementariamente se sugiere desarrollar nuevas investigaciones donde se obtengan resultados sobre la procedencia geográfica de escolares resilientes que terminaron como microempresarios exitosos, de la misma manera se sugiere el desarrollo de estudios de investigación de docentes magisteriales, docentes de educación superior, funcionarios de empresas públicas y privadas, que actualmente son exitosos, pero que en sus años de estudios escolares manifestaron factores de resiliencia.

Teniendo en cuenta que las hipótesis específicas planteadas han demostrado que contienen variables que tienen entre si un alto grado de significancia y coeficientes de correlación positivos. Los Factores de Resiliencia en el Educando, influyen significativamente en el éxito Microempresarial.

\section{REFERENCIAS BIBLIOGRÁFICAS}

Garmezy, N. (1991). Resiliency and vulnerability to adverse development al outcomer associated with poverty. American Behavioral Scientist. 34 (4) : 416 - 430
Katliorenco,M.A., Caceres,I.\& Fontecilla, M. (1997). Estado del arte en resiliencia. Washington. D.C., Organización Panamericana de la salud.

Peralta, s. Ramírez, A. y Buitrago, H. (2005). Factores resilientes asociados al rendimiento académico en estudiantes pertenecientes a la universidad de sucre. Tesis doctoral, Universidad de Sucre, Colombia.

Rutter, M. (1991). Recilience some conceptual considerationes. Trabajo presentado en Institutives Conferencias on Fostering Resilience, Washintong D.C.

Velásquez, C., \& Montgomery, W. (2009). Resiliencia y depresión en estudiantes de secundaria de Lima Metropolitana con y sin participación en actos violentos. Revista IIPSI, 12 (2), 75 - 87.

Villalva, P.M.A (2010). Factores de reciliencia asociados al rendimiento académico en estudiantes de contextos de alta vulnerabilidad social. Revista pedagógica 31 (88); 159 $-188$.

Werner, E. (1989). High risk children in joung adulthood: An longitudinal study from birt to 32 yeras. American Journal or the Psyquiatry. 59; 72 - 81 